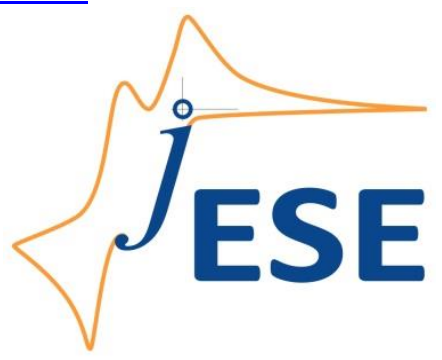

Open Access : : ISSN 1847-9286

www.jESE-online.org

Original scientific paper

\title{
Carbon nanofiber modified with osmium based redox polymer for glucose sensing
}

\author{
Amos Mugweru $^{1, 凶}$, Reaz Mahmud ${ }^{2}$, Kartik Ghosh², Adam K. Wanekaya ${ }^{3}$ \\ ${ }^{1}$ Department of Chemistry and Biochemistry, Rowan University, Glassboro, NJ 08028, USA \\ ${ }^{2}$ Physics Astronomy and Materials Science Department, Missouri State University, Springfield, \\ Missouri 65897, USA \\ ${ }^{3}$ Chemistry Department, Missouri State University, Springfield, Missouri 65897, USA \\ Corresponding authors E-mail: ${ }^{凶}$ mugweru@rowan.edu, Tel.: +1-856-2565454; Fax: +1-856-256-4478
}

Received: August 22, 2017; Revised: October 26, 2017; Accepted: November 12, 2017

\begin{abstract}
Electrochemical detection of glucose was performed on carbon nanofibers containing an osmium based redox polymer and using glucose oxidase enzyme. Redox polymer assembled on the nanofibers provided a more stable support that preserved enzyme activity and promoted the electrical communication to the glassy carbon electrode. The morphologies, structures, and electrochemical behavior of the redox polymer modified nanofibers were characterized by scanning electron microscope, energy dispersive spectrometer and voltammetry. The glucose oxidase showed excellent communication with redox polymer as observed with the increased activity toward glucose. Both cyclic voltammetry and amperometry showed a linear response with glucose concentration. The linear range for glucose determination was from 1 to $12 \mathrm{mM}$ with a relatively high sensitivity of $0.20 \pm 0.01 \mu \mathrm{A} \mathrm{mM}^{-1}$ for glucose oxidase in carbon nanofibers and $0.10 \pm 0.01$ $\mu \mathrm{A} \mathrm{mM} \mathrm{mM}^{-1}$ without carbon nanofibers. The apparent Michaelis-Menten constant $\left(K_{\mathrm{m}}\right)$ for glucose oxidase with carbon nanofibers was $0.99 \mathrm{mM}$. On the other hand, the $K_{\mathrm{m}}$ value for the glucose oxidase without the nanofibers was $4.90 \mathrm{mM}$.
\end{abstract}

Keywords

Cyclic voltammetry; Amperometry; Michaelis-Menten kinetics; Glucose sensors

\section{Introduction}

Regular monitoring of glucose concentration in the blood is a key in the diagnosis as well as treatment of diabetes. There is continuous interest in low cost, stable and sensitive biosensors for glucose. Many glucose sensing platforms have been developed using glucose oxidase enzyme and various forms of redox mediators on electrodes [1-4]. Glucose oxidase (GOX) is the most popular enzyme extensively used in fabrication of electrochemical glucose biosensor [5] as well as in other 
bio-catalytic systems [6,7]. Previously, osmium based redox polymer was used as a mediator entrapped together with GOX hydrogels through the UV-initiated free radical cross-linking [1,2].

The advantage of using electrochemical methods with glucose oxidase enzyme in glucose sensors is the fact that the enzyme is very selective to glucose. Like all enzymes, glucose oxidase stability is affected by the environment conditions such as $\mathrm{pH}$ and temperature [8]. Incorporating conducting nanomaterials as a transducer material provides fast and accurate electron transfer at electrode surface. The combination of nanomaterials and conducting polymers has attracted remarkable attention for development of new immobilization matrices for enzymes.

Many glucose sensors are incorporating carbon nanotubes for immobilization of different electron transfer mediators $[9,10]$. Carbon nanomaterials have excellent properties and unique structure for use in the study of direct electrochemistry of enzymes [11,12]. Enzyme immobilization techniques are the key in improving its performance. Recently, nanofibers have shown to be ideal in immobilization of enzymes $[13,14]$. Carbon nanofibers made from nylon have been used for biosensing applications due to their excellent stability and biocompatibility $[15,16]$. Nylon nanofibers have porous structure and have large surface area [17]. In general, the nanofibers are good materials for enzyme immobilization due to high loading resulted from high interfacial adhesion. Direct electron transfer (DET) between glucose oxidase enzyme and electrode surface would be ideal in glucose sensor. Then, the electron transfer process is quite sluggish [18] and hence use of mediators is initiated. Inclusion of mediators and carbon-based nanomaterials can greatly improve the electro-catalytic performance of these sensors. Enzymes, including GOX may denature upon absorption on the nanostructured surface. Denaturation of the enzyme may result in decreased or total loss of function as a result of altered structure [19]. Hybrid materials comprising of carbon nanofibers and redox polymer can induce a synergic effect of protecting the enzyme from the nanostructured surface, as well as providing a stable surface for immobilization onto a carbon surface. This can facilitate charge transfer from GOX to redox polymer, as well as increase of conductivity of the composite polymeric film. Nanofibers are promising nanomaterials as enzyme immobilization matrices [20].

In this work, poly[vinylpyridine Os(bipyridine) ${ }_{2}$ ] Cl (referred here as redox polymer) coupled with carbon nanofibers were used as materials in which GOX was immobilized. New literature suggests that this redox polymer can also mediate electron transfer between cells and electrodes [21]. The osmium based redox polymer has well-established redox chemistry and has been used before in different matrices [22-24]. The Os(II)/(III) redox potential is lowered when the osmium is complexed with ligands. We have previously used osmium redox polymers in construction of different biosensors $[25,26]$. The new material containing carbon nanofibers and osmium based redox polymers were used to fabricate a stable glucose sensor.

\section{Experimental}

\section{Materials and reagents}

Glucose oxidase (GOX, EC 1.1.3.4, Type X-S, 128 units/mg, solid from Aspergillus niger), hexafluorophosphate, sodium dithionite, ether, N,N-Dimethyl formamide, hydrochloric acid, ethyl alcohol, ethylene glycol, and acetonitrile were purchased from VWR (West Chester, PA). Pyrolytic graphite electrodes (area $0.07 \mathrm{~cm}^{2}$ ) were obtained from momentive performance materials Quarts Inc. The polishing alumina and $1 \mu \mathrm{m}$ diamond polish were obtained from Bioanalytical Systems Inc (West Lafayette IN). Graphitized carbon nanofibers free from iron, poly(4-vinyl pyridine) and poly(ethylene glycol) di-acrylate (MW 575), were purchased from Sigma-Aldrich, while hexa-fluoro- 
phosphate, sodium dithionite, ether, N,N-Dimethyl formamide, ammonium hexa-chloroosmate (IV) and 2-bromoethylamine hydro-bromide, were from Alfa Aesar.

\section{Synthesis of redox polymer poly[vinylpyridine Os(bipyridine) $\left.{ }_{2}\right] \mathrm{Cl}$}

The poly-cationic redox polymer, poly[4-vinylpyridine Os(bipyridine) $\left.{ }_{2}\right] \mathrm{Cl}$ (noted as Poly-BiPyOsCl) was synthesized according to a procedure described previously [27-29]. Os(bpy ${ }_{2} \mathrm{Cl}_{2}$ was synthesized according to a standard procedure with minor modifications [30]. Briefly, bipyridine $(1.44 \mathrm{~g})$ and ammonium hexa-chloroosmate (IV) $(2.0 \mathrm{~g})$ were mixed in $100 \mathrm{~mL}$ ethylene glycol before refluxing for one hour. Addition of a supersaturated solution of sodium dithionate to the reaction mixture precipitated $\mathrm{Os}(\mathrm{bpy})_{2} \mathrm{Cl}_{2}$. The product was repeatedly washed with water and finally with ether.

In synthesis of poly[vinylpyridine Os(bipyridine) $\left.{ }_{2}\right] \mathrm{Cl}, \mathrm{Os}(\mathrm{bpy})_{2} \mathrm{Cl}_{2}(0.988 \mathrm{~g})$ and poly(4-vinylpyridine) $(0.860 \mathrm{~g})$ were mixed and then heated to reflux under a nitrogen atmosphere to prevent oxidation. After two hours of reflux, the solution was cooled down to room temperature. $60 \mathrm{~mL}$ of DMF and $3.0 \mathrm{~g}$ of 2-bromoethylamine hydro-bromide were added and then stirred overnight at $50^{\circ} \mathrm{C}$. A crude polymer precipitate was formed by pouring the solution into rapidly stirred acetone. The soluble portion of the synthesized redox polymer was used in this work. The structure of this polymer is shown in Figure 1.

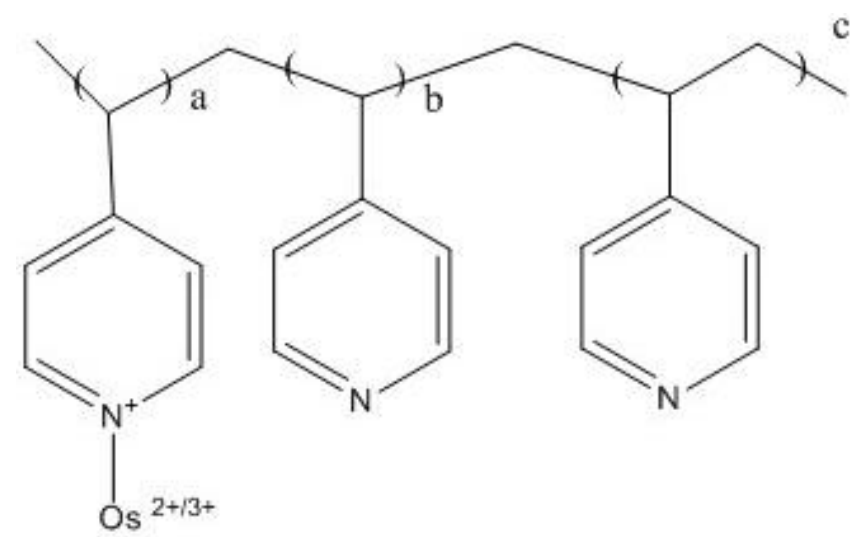

(bpy) ${ }_{2} \mathrm{Cl}$

Figure 1. Structure of poly[vinylpyridine Os(bipyridine) $\left.{ }_{2}\right] \mathrm{Cl}$ redox polymer, (Poly-BiPy-OsCl) $(a=1, b=4, c=1)$ are based on initial reaction conditions

\section{Electrochemical apparatus and preparation procedure}

Cyclic voltammetry (CV) and amperometric techniques were carried out with a computer controlled electrochemical workstation (CHI 660c, USA) with $98 \%$ ohmic drop (IR) compensation. A three-electrode electrochemical cell was used for all electrochemical experiments. The working and the counter electrodes were obtained from Bioanalytical Systems Inc (West Lafayette, IN). Glassy carbon electrodes $\left(0.07 \mathrm{~cm}^{2}\right)$ were polished with $1 \mu \mathrm{m}$ diamond polishing paste then ultra-sonicated in ethanol and distilled water successively for 1 min followed by rinsing in water.

A pyrolytic graphite (PG) electrode functionalized with composite materials of carbon nanofibers, osmium based redox polymer and glucose oxidase was used as the working electrode. Platinum wire was used as the counter electrode. $\mathrm{Ag} / \mathrm{AgCl}$, equipped with a glass tip, separated from the sample solution compartment by a salt-bridge containing $\mathrm{KCl}$ and terminating in a medium porosity glass frit, was used as the reference electrode. All electrochemical measurements were carried out at ambient conditions. The concentration of acetate buffers were $50 \mathrm{mM}$. A precursor solution $2 \mathrm{~mL}$ 
of $10 \mathrm{mg} / \mathrm{mL}$ redox polymer and $0.5 \mathrm{~mL}$ of $50 \mathrm{mg} / \mathrm{mL}$ GOX was made using the acetate buffer. This precursor solution was mixed with $0.5 \mathrm{~g}$ of carbon nanofibers (CNF) and then diluted to $10 \mathrm{ml}$ with acetate buffer. After mixing well, $10 \mu \mathrm{L}$ of this composite mixture was casted as dispersion on the electrode and left to dry at room temperature. Using the electrode area of $0.07 \mathrm{~cm}^{2}$, we can estimate the polymer loading to be $0.3 \mathrm{mg} / \mathrm{cm}^{2}$, glucose oxidase was $0.36 \mathrm{mg} / \mathrm{cm}^{2}$ while the CNF loading was $7.1 \mathrm{mg} / \mathrm{cm}^{2}$. The electrode containing redox polymer, CNF and GOX was later rinsed with water before use. The pictorial representation of the electrode preparation is shown in Figure 2.

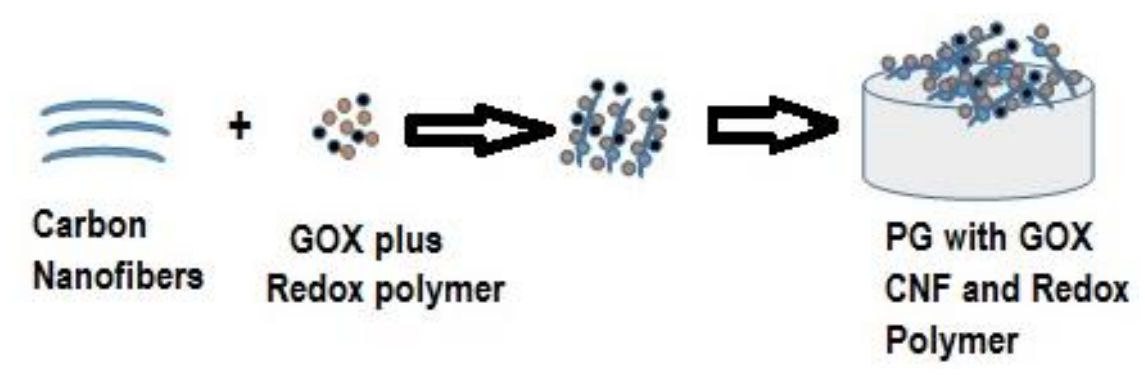

Figure 2. Schematic illustration of the preparation of electrode containing carbon nanofiberredox polymer-glucose oxidase, PG-CNF-(Poly-BiPy-OsCl)-GOX

\section{Results and discussion}

\section{Scanning electron microscopy and Energy-dispersive X-ray spectroscopy}

The scanning electron microscope images were recorded using a FEI Quanta 200 Scanning Electron Microscope with an Oxford Inca EDS detector. Figure 3 shows SEM images of redox polymer with GOX on CNF at different regions and different magnification.
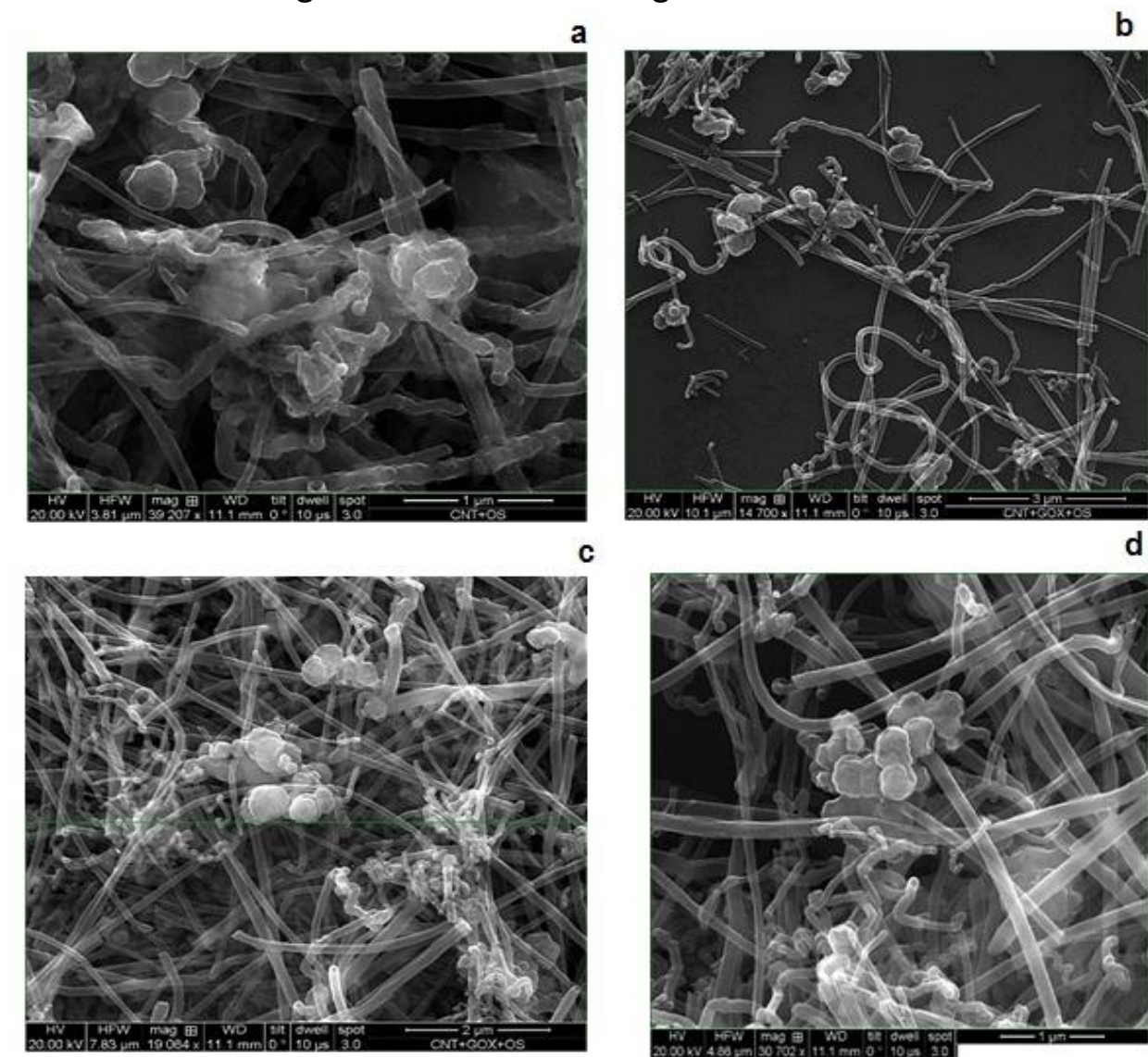

c

d

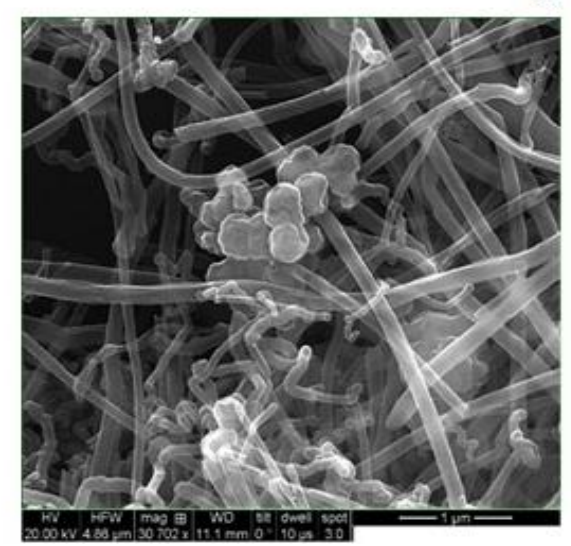

Figure 3. SEM images at different magnification of carbon nanofibers with redox polymer and glucose oxidase 
The graphitized conical CNF were used as the anchor for both, the redox polymer and the GOX. From Figure 3 we can estimate the length of the longest nanofiber to about $5 \mu \mathrm{m}$. Figure 3 a shows the CNF functionalized with the redox polymer. The redox polymer is not uniformly distributed on the CNF, but it is making some CNF to be bound closer together. Immobilization of the redox polymer on CNF results in formation of globular particles around the nanofibers (Figure 3a). Figures $3 b-d$ show images at various magnifications of CNF with both, the redox polymer and the GOX. It is obvious from these images, that the carbon nanofibers integrity was not affected. GOX adhered well onto carbon nanofibers after immobilization. However, the adherence of GOX to the nanofiber surface was not well-organized.

Figure 4 shows the energy-dispersive X-ray spectroscopy (EDX) of composite materials of CNF and the osmium based redox polymer. The presence of the osmium signal indicates that the polymer was incorporated into the composite material.

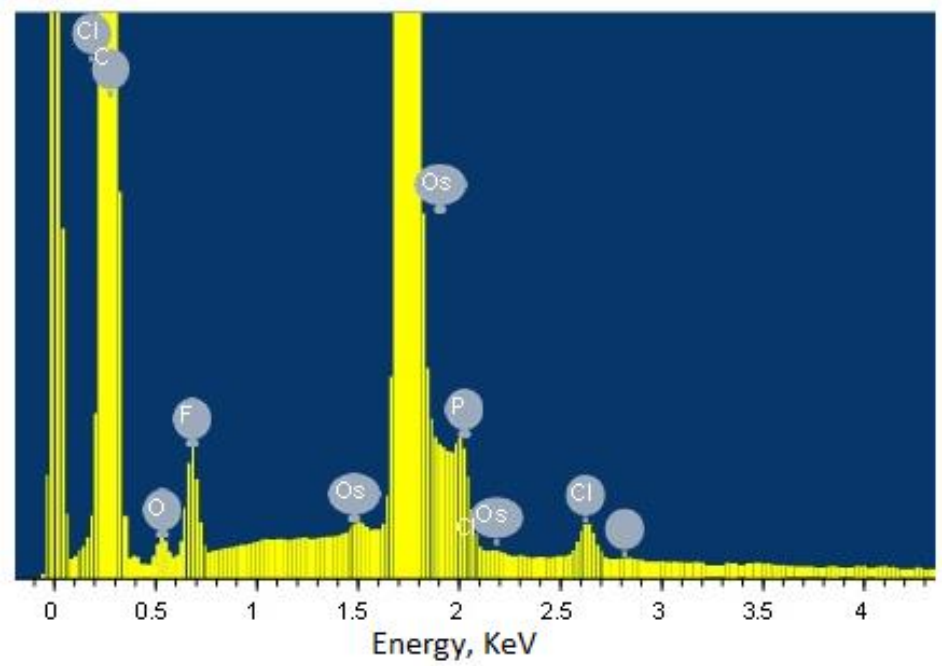

Figure 4. EDX spectrum of the redox polymer carbon nanofiber composite directly casted on the glassy carbon

\section{Cyclic voltammetry}

Figure 5 shows cyclic voltammograms of the redox polymer casted on a glassy carbon electrode with and without CNF. Reversible pairs of oxidation-reduction peaks correspond to the Os(II)/Os(III) redox couple.

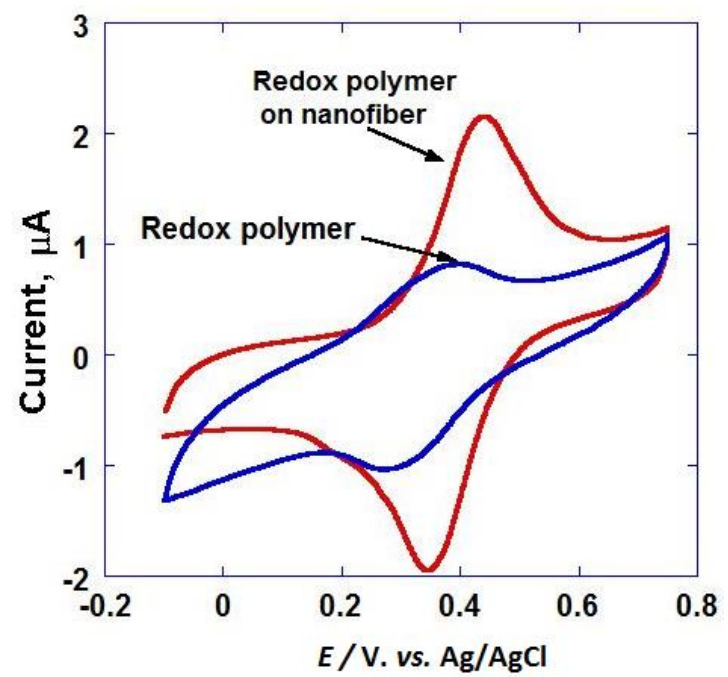

Figure 5. Cyclic voltammograms of redox polymer on glassy carbon electrode recorded at $50 \mathrm{mV} / \mathrm{s}$ scan rate in 50 mM acetate buffer, pH 5.5: (blue) (Poly-BiPy-OsCl); (red) CNF-(Poly-BiPy-OsCl) 
The redox polymer on CNF shows an enhanced signal compared with polymer electrode, what is seen as peak amplitudes that are about three times greater than those of the redox polymer by itself. However, incorporation of the redox polymer in the CNF resulted in a $0.05 \mathrm{~V}$ positive potential shift of the redox peak. The oxidation peak for redox polymer recorded at $50 \mathrm{mV} / \mathrm{s}$ scan rate occurred at about $0.44 \mathrm{~V}$, while the reduction peak occurred at $0.34 \mathrm{~V}$ using the CNF.

The oxidation peak for polymer by itself on the electrode surface appeared at $0.39 \mathrm{~V}$ while the reduction peak appeared at about $0.29 \mathrm{~V}$. In earlier literature, the redox polymer trapped in polyethylene glycol gel still gave similar voltammograms but the peak current was unstable after continuous scanning [31,32]. For the redox polymer modified nanofibers, scanning several times over three weeks yielded the same peak current, indicating thus a good stability of redox polymer adsorbed on CNF.

In presence of glucose the current using PG-CNF-(Poly-BiPy-OsCl)-GOX was found to increase (Figure 6). Presence of glucose caused the current increase. Flavin adenine dinucleotide of glucose oxidase GOX(FAD) reacts with $\beta$-D-glucose to form a reduced form $G O X\left(F A D H_{2}\right)$ and gluconic acid and hydrogen peroxide. The reduced form of $\mathrm{GOX}\left(\mathrm{FADH} \mathrm{H}_{2}\right)$ is in turn oxidized by the electrochemically generated $\mathrm{Os}^{3+}$ form of the redox polymer, setting up a catalytic pathway which produces an enhanced oxidation peak. The electrons are transferred from the enzyme to the redox polymer, shuttled between the redox sites in self exchange reaction until being transferred to an electrode surface. The catalytic current produced is proportional to the glucose concentration.
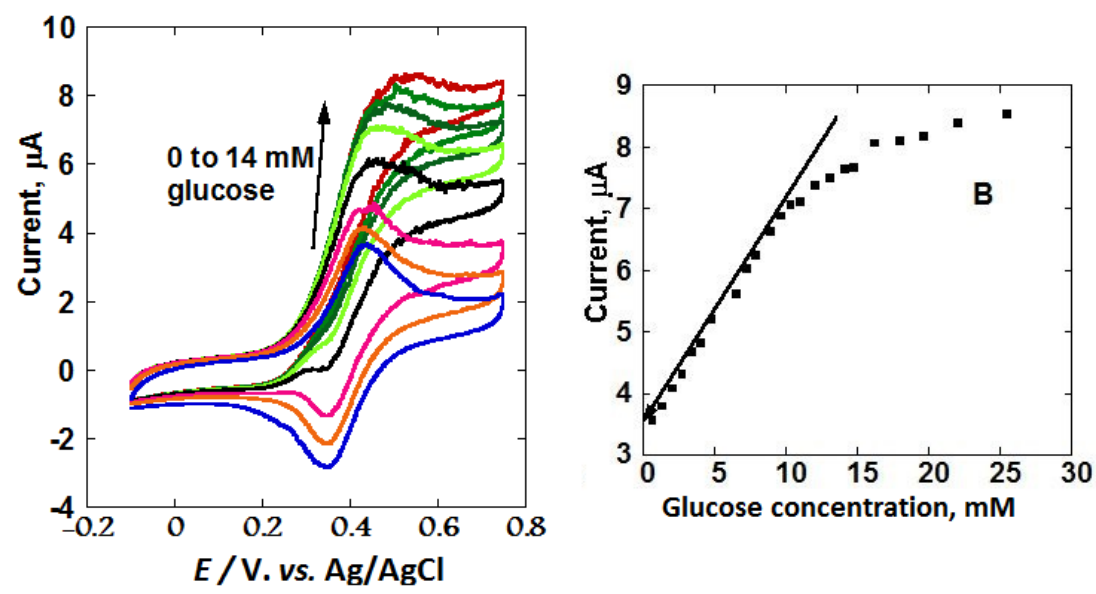

Figure 6. (a) Cyclic voltammograms of PG-CNF-(Poly-BiPy-OsCI)-GOX in $50 \mathrm{mM}$ acetate buffer, $\mathrm{pH}$ 5.5, recorded at $50 \mathrm{mV} \mathrm{s}^{-1}$ scan rate after continuous addition of glucose; (b) calibration curve for glucose

Figure 6a shows the cyclic voltammograms of PG-CNF-(Poly-BiPy-OsCl)-GOX system recorded after continuous addition of glucose. The resultant voltammogram in presence of glucose shows a significant increase in the magnitude of the oxidation peak. Figure $6 \mathrm{~b}$ shows the observed peak current values plotted against concentration. The glucose concentration shows a linear relationship with the peak current up to about $12 \mathrm{mM}$ of glucose. A plot of increase in the peak current versus the concentration of glucose yielded linear plot $(y=3.4+0.35 x, R=0.996, n=10)$ with $0.20 \pm 0.01$ $\mu \mathrm{A} \mathrm{mM} \mathrm{mM}^{-1}$ sensitivity. Glucose concentration higher than $12 \mathrm{mM}$ did not yield a current in the linear region. The linear response obtained in the range of 1 to $12 \mathrm{mM}$ is an improvement related to 0.05$100 \mu \mathrm{M}$ reported recently for comparable systems [33-35]. In the present system, presence of oxygen did not influence the rate of the reaction and hence the catalytic current is obtained. There was no difference in results obtained when oxygen was removed by bubbling the solution with nitrogen and blanketing the solution with nitrogen. 
PG-CNF-(Poly-BiPy-OsCl)-GOX and PG-(Poly-BiPy-OsCl)-GOX systems were also compared using the cyclic voltammetry results. The oxidation peak currents of both systems were recorded with increased amount of glucose in the reaction cell. The catalytic currents obtained for both systems at different glucose concentrations are compared in Figure 7. It is clear that presence of CNF in the system amplifies the catalytic current obtained using cyclic voltammetry.

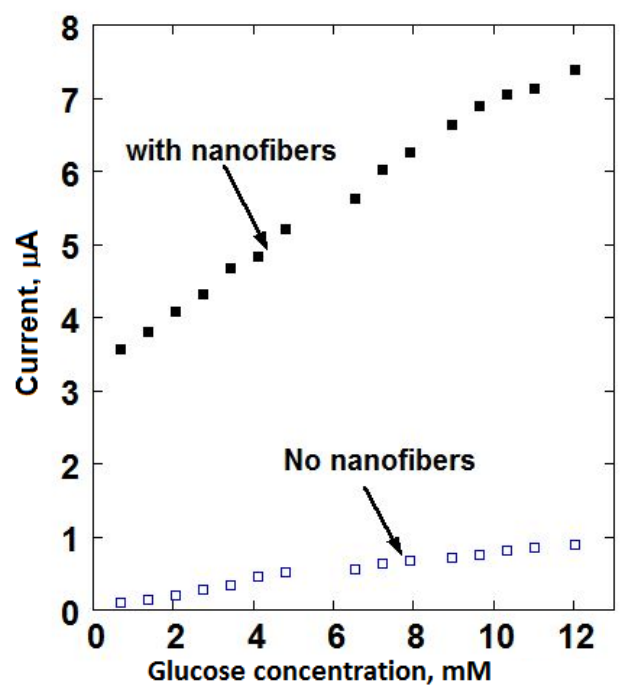

Figure 7. Comparison of glucose calibration curves for the electrode with nanofibers, PG-CNF-(Poly-BiPy-OsCl)-GOX and without nanofibers, PG-(Poly-BiPy-OsCl)-GOX

\section{Amperometry}

Figure 8a shows amperometric responses of both, PG-CNF-(Poly-BiPy-OsCl)-GOX and PG-(PolyBiPy-OsCl)-GOX electrodes. Each electrode was held at $0.35 \mathrm{~V} v \mathrm{vs} . \mathrm{Ag} / \mathrm{AgCl}$ and the current was continuously monitored as a function of time. For both systems, the amperometric responses showed a clear stepwise increase in the current value upon addition of glucose. However, the amperogram using PG-CNF-(Poly-BiPy-OsCl)-GOX shows a much higher current increase than PG(Poly-BiPy-OsCl)-GOX using the same amount of glucose.
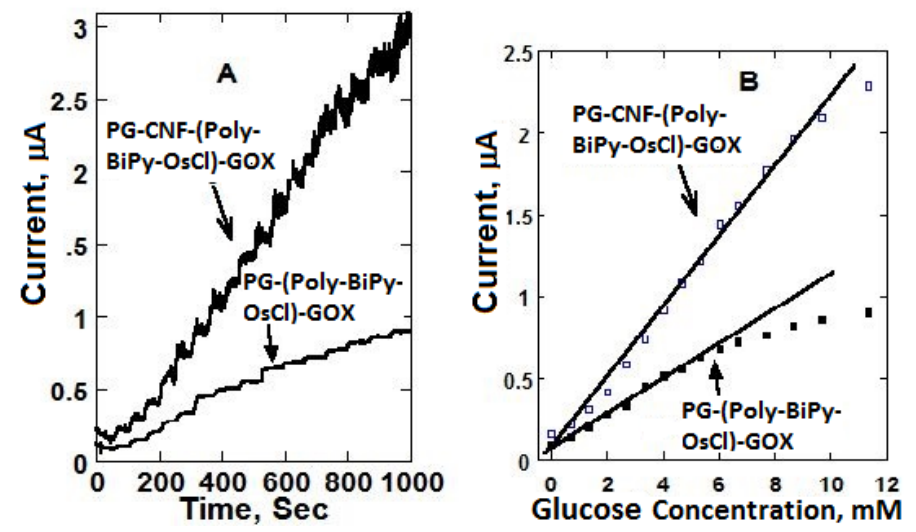

Figure 8. (a) Current versus time curve of PG-CNF-(Poly-BiPy-OsCl)-GOX and PG-(Poly-BiPy-OsCl)-GOX in $50 \mathrm{mM}$ acetate buffer, $\mathrm{pH} 5.5$, at $0.35 \mathrm{~V}$ vs. Ag/AgCl after continuous addition of glucose (b) Comparison of current versus glucose concentration for PG-(Poly-BiPy-OsCl)-GOX and PG-CNF-(Poly-BiPy-OsCl)-GOX sensors.

This could probably be due to a three-dimensional structure formed by anchoring the GOX on CNF. This three-dimensional structure coupled with the large surface area of CNF resulted in the high sensitivity observed. Glucose oxidase is comprised of two identical subunits. The two units have 
two moles of flavin adenine dinucleotide (FAD) which are located deep inside the enzyme [36]. For our sensor to provide immediate response, analytes have to move into the GOX and electrons have to move into the redox polymer. The rapid response observed imply that this movement is not hindered at the same time as the CNF containing the redox polymer must remain in place. Each step current observed represents an increase of $0.70 \mathrm{mM}$ of glucose concentration. A plot of change of current versus the glucose concentration also yielded a linear plot (Figure 8b). Higher sensitivity of PG-CNF-(Poly-BiPy-OsCl)-GOX sensor compared to PG-(Poly-BiPy-OsCl)-GOX was observed.

The sensitivity of electrodes with carbon nanofibers was $0.21 \pm 0.01 \mu \mathrm{A} \mathrm{mM}{ }^{-1}$, while the sensitivity of electrodes without the carbon nanofibers was $0.10 \pm 0.01 \mu \mathrm{A} \mathrm{mM}{ }^{-1}$. The use of CNF improves the sensitivity of this sensor by around fifty percent. The sensitivity of the current sensor is much lower compared with others recently reported [37,38]. For the system with CNF, the peak current plateaued at about $12 \mathrm{mM}$ glucose concentration, while without CNF, the peak current plateaued at about $6 \mathrm{mM}$.

Glucose oxidase is known to have a short operational life due to the lack of stability. This is a major drawback in the construction of glucose biosensors [39]. To compare the enzymatic glucose metabolism in PG-CNF-(Poly-BiPy-OsCl)-GOX and PG-(Poly-BiPy-OsCl)-GOX systems, the MichaelisMenten kinetics were determined and compared. Based on the equations (1) and (2) and using the plots drawn in Figure $9, I_{\max }$ and Michaelis-Menten constant $\left(K_{\mathrm{m}}\right)$ values for the GOX were calculated for both systems.

$$
\begin{aligned}
& I_{\mathrm{ss}}=\frac{I_{\max } C_{\text {glucose }}}{K_{m}+C_{\text {glucose }}} \\
& \frac{1}{I_{\mathrm{ss}}}=\frac{1}{I_{\max }}+\frac{K_{\mathrm{m}}}{I_{\max }} \frac{1}{C_{\text {glucose }}}
\end{aligned}
$$

In equations (1) and (2), Imax represents the maximum current achieved in the system, while the $I_{s s}$ is the steady state current.

Figure 9 shows the Lineweaver-Burk plots of PG-CNF-(Poly-BiPy-OsCl)-GOX and PG-(Poly-BiPyOsCl)-GOX, respectively. The apparent $K_{\mathrm{m}}$ values with respect to glucose were estimated using the eq. (2) and plots in Figure 9.

These enzymatic kinetics studies showed that PG-CNF-(Poly-BiPy-OsCl)-GOX system had a $K_{m}$ of $0.99 \mathrm{mM}$, while the calculated $K_{\mathrm{m}}$ value for PG-(Poly-BiPy-OsCl)-GOX was $4.90 \mathrm{mM}$. It is obvious that glucose oxidase enzyme catalytic property of PG-CNF-(Poly-BiPy-OsCl)-GOX is much higher than of PG-(Poly-BiPy-OsCl)-GOX enzymes. Mass transfer limitations of glucose is the same in both electrodes and the only explanation for higher activity at CNF could be due to a loss of glucose oxidase activity in PG-(Poly-BiPy-OsCl)-GOX electrode. This might be due to the conformation change of the enzyme in this system. For most enzymes a favorable environment is essential for the optimum enzyme activity. Our results indicate an extraordinary stability of the PG-CNF-(Poly-BiPyOsCl)-GOX system as compared to PG-(Poly-BiPy-OsCl)-GOX.

The recent literature showed that $K_{\mathrm{m}}$ for glucose oxidase immobilized at other materials is equal to $2.84 \mathrm{mM}$ [40]. Here obtained value of the Michaelis-Menten constant of $0.99 \mathrm{mM}$ is much lower than $5.20 \mathrm{mM}$ for GOX/gold-platinum alloy nanoparticles/carbon nanotubes/chitosan system [41] and also $1.42 \mathrm{mM}$ for GOX/PVA- $\mathrm{Fe}_{3} \mathrm{O}_{4} / \mathrm{Sn}$ [42]. Our experiment was performed in the acetate buffer of $\mathrm{pH} 5.5$ which is slightly different from the optimum $\mathrm{pH}$ for GOX activity. However, this $\mathrm{pH}$ is optimum for redox polymer mediator. 

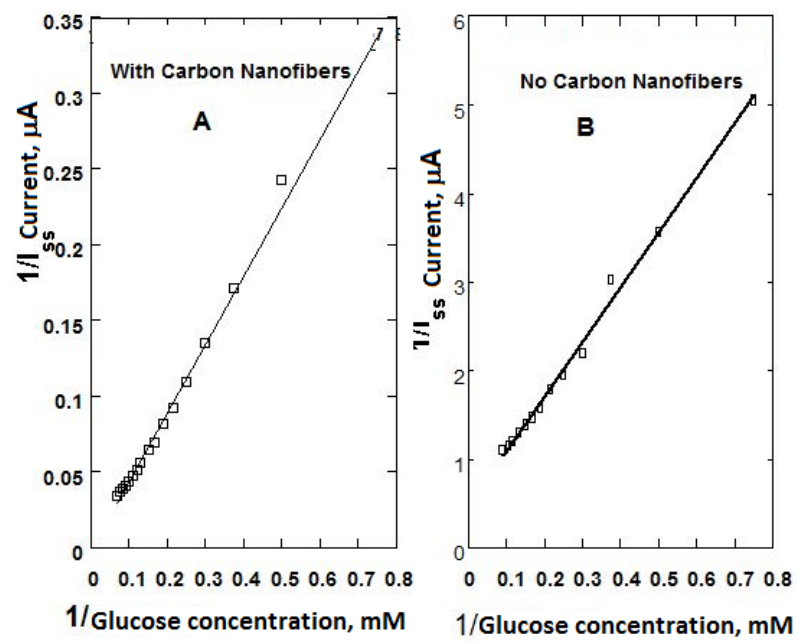

Figure 9. Lineweaver-Burk plots for glucose oxidase (a) PG-CNF-(Poly-BiPy-OsCl)-GOX;

(b) PG-(Poly-BiPy-OsCl)-GOX

The ability of a sensor to discriminate other interfering species with similar properties to the target analyte is very important. Oxidative species such as uric acid, fructose, sodium chloride and sucrose among others co-exist with glucose in human blood. The interference study was carried using amperometry by spiking $1.4 \mathrm{mM}$ of each of the interfering agent in the electrolyte. The resulting amperogram for PG-CNF-(Poly-BiPy-OsCl)-GOX is shown in Figure 10.

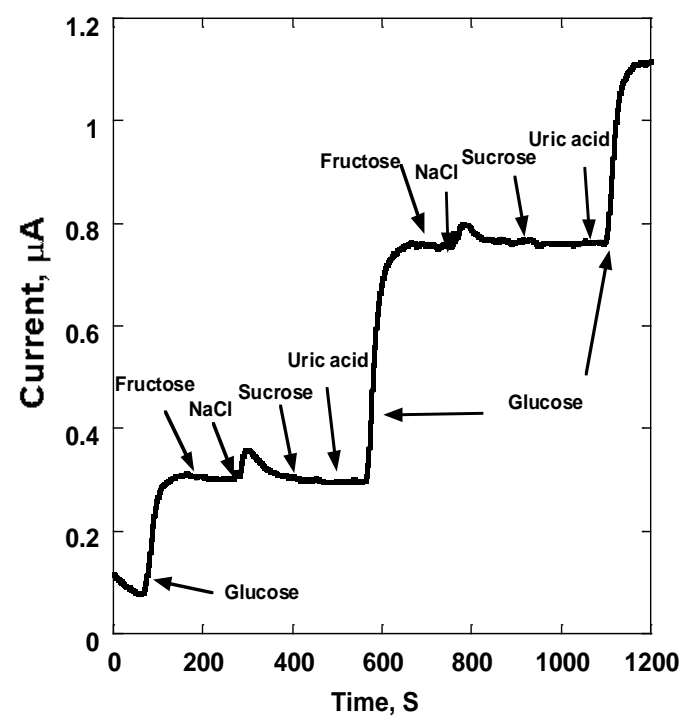

Figure 10. Current versus time curve of PG-CNF-(Poly-BiPy-OsCl)-GOX in $50 \mathrm{mM}$ acetate buffer, $\mathrm{pH} 5.5$ at $0.35 \mathrm{~V} v$ s. $\mathrm{Ag} / \mathrm{AgCl}$ after continuous addition of glucose, fructose, sodium chloride sucrose and uric acid

The peak current obtained from glucose solution and interfering ions was noted and it proves that glucose oxidation current was dominant when compared to oxidation currents of uric acid, fructose, sucrose and sodium chloride. The small rise with addition of sodium chloride suggests the electrolyte solution may not have been properly buffered. In general, the PG-CNF-(Poly-BiPy-OsCl)GOX sensor shows high selectivity towards glucose.

\section{Conclusions}

In this work, carbon nanofibers were used in conjunction with poly[vinylpyridine $\mathrm{Os}$ (bipyridine $)_{2} \mathrm{Cl}$ to prepare a sensor for glucose determination. Both, the glucose oxidase and the 
osmium based redox polymer adhered strongly onto the carbon nanofibers as observed from SEM images. In this new system, glucose oxidase was found to be almost two fold active and more stable, using both cyclic voltammetry and amperometry techniques. The Michaelis-Menten constant $\left(K_{\mathrm{m}}\right)$ and steady state current $\left(I_{s s}\right)$ values showed the improved GOX performance in presence of carbon nanofibers, what is partly due to enhanced electron transfer from the glucose oxidase to the redox polymer. If properly optimized, the new material could be promising in improving glucose biosensors involving glucose oxidase enzymes.

Acknowledgements: The authors would like to thank the Zahilis Mazzochette and the department of Chemistry and Biochemistry, Rowan University.

\section{References}

[1] A. Mugweru, B. L. Clark, M. V. Pishko, Electroanalysis 19 (2007) 453-458.

[2] A. Mugweru, B. L. Clark, M. V. Pishko, Journal of Diabetes Science and Technology 1 (2007) 366-371.

[3] A. Heller, Current Opinion in Chemical Biology 10 (2006) 664-672.

[4] P. Åsberg, O. Inganäs, Biosensors \& Bioelectronics 19 (2003) 199-207.

[5] Y.-T. Wang, L. Yu, Z.-Q. Zhu, J. Zhang, J.-Z. Zhu, C.-H. Fan, Sensors and Actuators B: Chemical 136 (2009) 332-337.

[6] A. Heller, Physical Chemistry Chemical Physics 6 (2004) 209-216.

[7] S. Cosnier, A. Le Goff, M. Holzinger, Electrochemistry Communications 38 (2014) 19-23.

[8] L.M. Lu, L. Zhang, F.L. Qu, H.X. Lu, X.B. Zhang, Z.S. Wu, S.Y. Huan, Q.A. Wang, G.L. Shen, R.Q. Yu, Biosensors and Bioelectronics 25 (2009) 218-223.

[9] S. Xi, Sensors and Actuators A: Physical 198 (2013) 15-20.

[10] M. Rezaeinasab, Electroanalysis 29 (2017) 423-432.

[11] X. Zhang, D. Liu, L. Li, T. You, Scientific Reports 5 (2015) 9885.

[12] S.-G. Hong, Biotechnology and Bioprocess Engineering 21 (2016) 573-579.

[13] H. Liang, S. Jiang, Q. Yuan, G. Li, F. Wang, Z. Zhang and J. Liu, Nanoscale 8 (2016) 6071-6078.

[14] D. Liu, X. Zhang, T. You, ACS Applied Materials \& Interfaces 6 (2014) 6275-6280.

[15] M. Scampicchio, A. Arecchi, A. Bianco, A. Bulbarello, C. Bertarelli, S. Mannino, Electroanalysis 22 (2010) 1056-1060.

[16] M. Yasuzawa, Y. Omura, K. Hiura, J. Li, Y. Fuchiwaki, M. Tanaka, Analytical Sciences 31 (2015) 11111114.

[17] A. Arecchi, M. Scampicchio, S. Drusch, S. Mannino, Analytica Chimica Acta 659 (2010) 133-136.

[18] Y. Wang, L. Liu, M. Li, S. Xu, F. Gao, Biosensors \& Bioelectronics 30 (2011) 107-111.

[19] A. Seehuber, R. Dahint, The Journal of Physical Chemistry B 117 (2013) 6980-6989.

[20] S. Huang, Y. Ding, Y. Liu, L. Su, R. Filosa, Y. Lei, Electroanalysis 23 (2011) 1912-1920.

[21] G. Pankratova, K. Hasan, D. Leech, L. Hederstedt, L. Gorton, Electrochemistry Communications 75 (2017) 56-59.

[22] M. Grattieri, ChemElectroChem, 3 (2016) 1884-1889.

[23] Y. Nakabayashi, A. Omayu, S. Yagi, K. Nakamura, J. Motonaka, Analytical Sciences 17 (2001) 945-950.

[24] M. Kirthiga, Electrochimica Acta 230 (2017) 89-97.

[25] A. Mugweru, B. Wang, J. Rusling, Analytical Chemistry 76 (2004) 5557-5563.

[26] M. Amos, Analytical Sciences 24 (2008) 1105-1110.

[27] B. A. Gregg, A. Heller, The Journal of Physical Chemistry 95 (1991) 5970-5975.

[28] V. Soukharev, N. Mano, A. Heller, Journal of the American Chemical Society 126 (2004) 8368-8369.

[29] F. Mao, N. Mano, A. Heller, Journal of the American Chemical Society 125 (2003) 4951-4957.

[30] P. Denisevich, H.D. Abruna, C.R. Leidner, T.J. Meyer, R.W. Murray, Inorganic Chemistry 21 (1982) 2153-2161.

[31] A. Mugweru, Electroanalysis 19 (2007) 453-458.

[32] A. Mugweru, Journal of Diabetes Science and Technology 1 (2007) 366.

[33] D. Xu, Analytical Biochemistry 489 (2015) 38.

[34] T. Y. Tekbasoglu, T. Soganci, M. Ak, A. Koca, M. K. Sener, Biosensors \& Bioelectronics 87 (2017) 81-88. 
[35] H. Wang, Sensors and Actuators: B, Chemical 216 (2015) 298-306.

[36] H. J. Hecht, H. M. Kalisz, J. Hendle, R. D. Schmid, D. Schomburg, Journal of Molecular Biology 229 (1993)153-172.

[37] L. Wang, J. Yu, Y. Zhang, H. Yang, L. Miao, Y. Song, ACS Applied Materials \& Interfaces 9 (2017) 90899095.

[38] J. Chen, Journal of the Electrochemical Society 164 (2017) B66-B73.

[39] M. Altikatoglu, Y. Basaran, C. Arioz, A. Ogan, H. Kuzu, Applied Biochemistry and Biotechnology 160 (2010) 2187-2197.

[40] V. Buk, Materials Science \& Engineering: C, Materials for Biological Application 74 (2017) 307-314.

[41] X. Kang, Z. Mai, X. Zou, P. Cai, J. Mo, Analytical Biochemistry 369 (2007) 71-79.

[42] Sanaeifar, M. Rabiee, M. Abdolrahim, M. Tahriri, D. Vashaee, L. Tayebi, Analytical Biochemistry 519 (2017) 19-26.

(C2017 by the authors; licensee IAPC, Zagreb, Croatia. This article is an open-access article distributed under the terms and conditions of the Creative Commons Attribution license (http://creativecommons.org/licenses/by/4.0/) 